\title{
Effets Du Labour Mécanisé Successif Sur Le Statut Nutritif Du Sol Et Le Rendement Du Riz Pluvial Dans La Commune d'Abomey Calavi (Sud Bénin)
}

\author{
Atidegla C.S. \\ Sintondji L. $O$. \\ Faculté des Sciences Agronomiques (FSA), Cotonou, Bénin \\ Hounkpe $J$. \\ Faculté des Sciences et Techniques (FAST), Cotonou, Bénin \\ Kpadonou $\boldsymbol{E}$. \\ Faculté des Sciences Agronomiques (FSA), Cotonou, Bénin
}

Doi: 10.19044/esj.2017.v13n30p341 URL:http://dx.doi.org/10.19044/esj.2017.v13n30p341

\begin{abstract}
The effects of mechanized plowing with power tiller and rotavator and that of the succession of production campaigns on the nutritive status of soil and the rice yield in pluvial system were studied in the low land of Dokomey in South of Benin. The methodological approach is based on field trials conducted during three successive production campaigns from 2014 to 2016 according to a split plot design with one factor (plowing).. Sampling and analysis of the physical and chemical soils' parameters were done at the beginning and end of each campaign and also the weighing of harvested paddy. Results showed that the fertility of the plots which received mechanized plowing and that of their yields increased noticeably during each campaign and along all the campaigns than those which received manual plowing. For example, from campaign 1 to 3 , the yields under mechanized plowing increased respectively of 35\%,37\% and 39\% more than those obtained under manual plowing. The same report was obtained for soils parameters where the contents of organic matters $(\mathrm{OM})$, nitrogen $(\mathrm{N})$, ratio $\mathrm{C} / \mathrm{N}$, cationic exchange capacity, assimilable phosphorus (Pass) and clay increased respectively of $31,63 \% ; 11,77 \% ; 13,39 \% ; 10,28 \% ; 6,03 \%$ et $3,74 \%$.Moreover, the correlations study between yield and nutritive status of soil showed likewise a high dependence between those parameters and the obtained yields. This dependence was highly significant at the level of $0.1 \%$ for th mechanized plowing.
\end{abstract}


Keywords: Fertility, nitrogen, organic matters, ratio $\mathrm{C} / \mathrm{N}$, clay, power tillerrotavator

\section{Résumé}

Les effets du labour mécanisé avec l'attelage motoculteur-rotavator ainsi que ceux de sa succession sur le statut nutritif du sol et sur le rendement du riz en régime pluvial ont été étudiés dans le bas-fond de Dokomey au Sud-Bénin. L’approche méthodologique utilisée est basée sur des essais agronomiques conduits pendant trois campagnes successives de production de 2014 à 2016 selon un dispositif en split-plot à un facteur (labour). Il a été procédé à des analyses des paramètres physico-chimiques du sol avant le démarrage et à la fin de chaque campagne ainsi qu'à la pesée des récoltes du riz paddy. Les résultats ont montré que la fertilité des parcelles en labour mécanisé ont des rendements qui ont sensiblement augmenté au cours de chaque campagne que ceux obtenus en labour manuel. Par exemple, de la campagne 1 à 3 , les rendements en labour mécanisé ont respectivement augmenté de 35, 37 et $39 \%$ de plus que ceux obtenus en labour manuel. De même les teneurs des paramètres du sol que sont: matières organiques, azote, rapport $\mathrm{C} / \mathrm{N}$, capacité d'échange cationique, phosphore assimilable et argile ont respectivement augmenté de 31,63\% ; $11,77 \% ; 13,39 \% ; 10,28 \% ; 6,03 \%$ et $3,74 \%$. En outre, l'étude des corrélations entre le rendement et le statut nutritif du sol a également montré qu'il y a une forte dépendance entre ces paramètres et les rendements obtenus. Cette dépendance est hautement significative au seuil de $0,1 \%$ pour le labour mécanisé.

Mots-clés : Fertilité, azote, matières organiques, rapport $\mathrm{C} / \mathrm{N}$, argile, motoculteur-rotavator

\section{Introduction}

Dans la plupart des pays au Sud du Sahara, le secteur agricole constitue un pilier fondamental du développement rural. Il est reconnu que l'augmentation de la production agricole est un précurseur essentiel permettant le passage d'une agriculture de subsistance à celle commerciale, synonyme d'amélioration des revenus des agriculteurs et de leur niveau de vie (FAO, 1988). La mise en valeur agricole des plaines inondables et des bas-fonds constitue l'une des réponses possibles à la crise actuelle des systèmes traditionnels de production (Sossa, 2001). C'est ce qui justifie aujourd'hui, la prise d'assauts de ces zones par les paysans béninois pour la culture du riz avec l'appui de nombreux programmes de développement et d'Organisations Non Gouvernementales (ONG) internationales et nationales. Sur une surface de plus de 150 millions d'hectares cultivés en riz dans le 
monde, environ 30 millions d'hectares sont occupés par le riz pluvial strict (FAO, 2009). En Amérique latine, sur un total de 8,2 millions d'hectares, 5,9 millions sont cultivés en riz pluvial, soit 78 pour cent de la surface totale (IRAT, 1984; Gonzales, Umali, 1985). En effet dans notre pays, le riz est une des cultures vivrières la plus produite. Il représente actuellement au niveau national, la $3^{\text {ème }}$ céréale en termes de production, après le maïs et le sorgho et il est le deuxième produit le plus consommé après le maïs (Kokoye, 2013). Apprécié pour ses qualités culinaires, le riz a pris une grande importance dans l'alimentation des ménages. Il est devenu la denrée de choix des populations. La demande de riz par personne est estimée à 20 kg par an et augmente de $6 \%$ par an (INRAB, 2005). L'enjeu est donc grand car le riz fait partie des quatre (04) filières prioritaires du Plan Stratégique de Relance du Secteur Agricole (MAEP, 2010) compte tenu de son importance socio-économique et alimentaire. En effet, il occupe au niveau national la troisième place en termes de production de céréales après le maïs et le sorgho et représente la deuxième céréale en termes de consommation après le maïs (Assigbe, 2002). Apprécié pour ses qualités culinaires, le riz a pris une grande importance dans l'alimentation des ménages mais il fait face pour sa culture à d'importantes contraintes liées à sa production qui repose à $84 \%$ sur le travail manuel (MAEP, 2011).

L'amélioration de la production agricole en général et celle du riz en particulier, ne peut être garantie que par l'adoption des facteurs de production dont il faut citer la mécanisation. En effet, il est très difficile d'imaginer actuellement un secteur agricole se développer sans une technologie améliorée. D'après FAO (2016), il ne fait aucun doute que la mécanisation agricole destinée à la multitude de petits exploitants agricoles en Afrique subsaharienne (ASS) a été une question négligée depuis trop longtemps. L'application de l'énergie agricole aux machines, au matériel et aux outils appropriés (la «mécanisation agricole») constitue un intrant agricole essentiel potentiellement capable de transformer les moyens de subsistance de millions de familles en milieu rural en facilitant la production de produits à plus forte valeur tout en éliminant la pénibilité physique liée aux pratiques agricoles fondées sur l'énergie musculaire. Il est donc établi que malgré que le rendement des cultures dépende de multiples facteurs, la mécanisation agricole constitue l'élément le plus important.

Plusieurs études à travers le monde ont démontré le rôle de la technologie sur le rendement des cultures (Mouzou, 1999). C'est pourquoi, dans le but de remédier à quelques unes des difficultés liées aux travaux manuels que des producteurs au Sud-Bénin ont entrepris la mécanisation de la culture du riz à la faveur de l'avènement en 2008-2014 du Programme pour la Promotion de la Mécanisation Agricole (PPMA) qui offrait les équipements à prix subventionnés et à crédit. Ainsi, grâce à ce programme, 
certains riziculteurs dont notamment ceux de Dokomey dans la Commune d'Abomey-Calavi ont acquis des équipements agricoles parce qu'ils disposent d'une vaste étendue de bas-fond avec un potentiel de réseau hydrographique important pour la culture du riz. C'est le cas de la zone d'étude couvrant l'arrondissement de Yèvié où l'introduction des techniques culturales modernes de production comme le labour mécanisé est la bienvenue dans certaines de ses exploitations en vue d'accrôitre les emblavures et le rendement. Dans la zone d'étude, les producteurs de Dokomey, pratiquent selon leurs capacités financières, le labour manuel avec la houe ou le labour mécanisé avec l'attelage motoculteur et rotavator car le choix du matériel de labour doit être raisonné en fonction des caractéristiques liées au sol, au climat et au matériel végétal car un sol bien structuré permet une bonne germination des graines et un bon développement racinaire des plantes (Girard, 2005). En plus, le travail du sol vise l'enfouissement des débris végétaux, l'ameublissement de la couche arable, la formation du lit de semence, la répartition de la terre fine et des mottes, la maîtrise de la propagation des mauvaises herbes, des parasites et des maladies, l'incorporation des engrais, etc. (Seguyl, 1994). C'est dans ce cadre que s'insère l'objectif de la présente étude qui vise à analyser les effets des deux types de labour (labour manuel et labour mécanisé) et de leur succession sur l'état nutritif du sol et le rendement du riz pluvial dans le basfond de Dokomey au Sud-Bénin.

\section{Materiel et methodes}

\section{Zone d'étude}

D'une superficie de $539 \mathrm{Km}^{2}$, la commune d'Abomey-Calavi qui comprend la zone d'étude est située dans le Département de l'Atlantique tandis que le village d'étude appelé Dokomey est localisé dans l'arrondissement de Zinvié. Comme toute la région méridionale, AbomeyCalavi jouit d'un climat de type sub-équatorial caractérisé par deux saisons de pluie qui sont alternées par deux saisons sèches à durées inégales se répartissant comme suit : i) une grande saison pluvieuse de Mars à Juillet, ii) une petite saison sèche en Août, iii) une petite saison pluvieuse de Septembre à Octobre et iv) une grande saison sèche de Novembre à Mars. La moyenne pluviométrique annuelle est de $1137 \mathrm{~mm}$ et la température moyenne annuelle est de $27,2^{\circ} \mathrm{C}$.

La Commune d'Abomey-Calavi se trouve sur deux bassins versants dont près des $3 / 4$ de la superficie $\left(433 \mathrm{~km}^{2}\right)$ sont drainés vers le fleuve Ouémé et le reste $166 \mathrm{~km}^{2}$ ) s'écoule vers le fleuve Zou. La frontière entre les Communes de Sô-Ava et d'Abomey-Calavi est la rivière Sô. D'une longueur de $84,4 \mathrm{~km}$, la rivière Sô fait partie des défluents du fleuve Ouémé (PDC, 2012). Ses plus forts débits sont observés pendant les crues de l'Ouémé qui 
s'installent entre le mois d'Août et de septembre (SCDA, 2012). Le périmètre rizicole de Dokomey étant contigu à la vallée de Sô, le débordement de cette dernière en période de crue provoque sa submersion. De façon concrète, le réseau hydrographique est constitué essentiellement de trois (3) plans d'eau que sont le lac Nokoué, la lagune côtière et la rivière Sô. Par ailleurs, la commune dispose d'une façade maritime juxtaposée à la lagune côtière, des marais, des ruisseaux et des marécages. Tout cela lui offre des potentialités touristiques et halieutiques.

Selon le zonage agro écologique réalisé au niveau communal (SCDA, 2009), Dokomey fait partie de la zone 3 des dépressions caractérisée par des sols ferralitiques et des sols hydromorphes. Les sols ferralitiques sont profonds, rouges de type argilo-limoneux, assez équilibrés dans leur texture qui tolère la culture d'un grand ensemble de spéculations végétales.

\section{Approche méthodologique \\ Tests agronomiques}

Les essais ont été conduits sur trois campagnes successives de production du cycle de Mai à Août de 2014 à 2016 sur le périmètre rizicole de Dokomey caractérisé par un sol argilo-limoneux. Le matériel végétal utilisé est le riz, variété IR841 qui est une variété intra spécifique issue de Oryza Sativa et les divers entretiens (fertilisations, désherbages et chasse aviaire) ont été identiques dans toutes les parcelles élémentaires. Il s'agit de deux sarclages ( 25 à 40 jours environ après le repiquage pour le $1^{\text {er }}$ et 60 à 70 jours après le $1^{\mathrm{er}}$ ), de la fumure de fond avec $200 \mathrm{~kg} / \mathrm{ha}$ d'engrais minéral NPK le jour du semis et $75 \mathrm{~kg} / \mathrm{ha}$ d'urée à l'apparition des panicules comme engrais de couverture. Quand à la chasse aviaire, elle est exécutée à partir de la floraison, à un mois de la récolte.

Les effets du labour mécanisé avec l'attelage motoculteur-rotavator sont testés sur les parcelles (avec emplacement fixe) du périmètre rizicole de Dokomey selon un dispositif en split-plot à un facteur (labour) et trois répétitions. Il s'agit de trois blocs de trois parcelles élémentaires par traitement en trois répétitions. Le facteur labour comporte les trois modalités que sont: «sans labour», «labour mécanisé» et «labour manuel». La surface de la parcelle élémentaire est $625 \mathrm{~m}^{2}$ et les dimensions sont $25 \mathrm{~m}$ x $25 \mathrm{~m}$. Les labours manuel et mécanisé sont réalisés sur une profondeur de $25 \mathrm{~cm}$.

Les variables mesurées concernent le statut nutritif du sol regroupant ses caractéristiques physico-chimiques (avant et après l'essai) et les rendements du riz. Les paramètres recherchés pour le sol sont: la granulométrie, le carbone organique $(\mathrm{C} \%)$, l'azote total $(\mathrm{N} \%)$, les cations (potassium $\mathrm{K}^{+}$, calcium $\mathrm{Ca} 2+$, magnésium $\mathrm{Mg} 2+$ et sodium $\mathrm{Na}+$ ), la capacité d'échange cationique (CEC), le phosphore assimilable (Pass) et le $\mathrm{pH}$ eau. 
Les mesures du rendement du riz ont concerné seulement le poids du paddy qui est pesé à la récolte.

\section{Prélèvements et analyses des échantillons du sol au laboratoire}

Les analyses des échantillons de sols sont effectuées au Laboratoire des Sciences du Sol de la Faculté des Sciences Agronomiques (FSA) de l'Université d'Abomey-Calavi (UAC).

Les prélèvements de sols ont été faits 2 fois sur les différentes parcelles au cours de la campagne. Le premier, avant le semis c'est-à-dire au cours de la préparation des parcelles élémentaires et le second après la récolte du riz. Cinq points de prélèvement ont été fixés au hasard sur les diagonales dans de chaque parcelle élémentaire pour l'échantillonnage du sol. Au niveau de chaque point, à l'aide d'une tarière, un échantillon de sol a été prélevé à $40 \mathrm{~cm}$ de profondeur et mélangés dans un seau. Cinq cent grammes de ce mélange ont été versés dans un sachet plastic étiqueté et envoyés au Laboratoire des Sciences du Sol de la FSA/ UAC, pour en déterminer les propriétés chimiques. La granulométrie par tamisage selon la loi de Stockes $(0-20 \mathrm{~mm})$ et sédimentation, la matière organique et le carbone ont été déterminés par la méthode de Walkley et Black, l'azote par la méthode de Kjeldahl, la CEC par la méthode Metson et le phosphore assimilable par la méthode de Bray et Kurtz. Enfin, les bases échangeables $\left(\mathrm{Ca}^{2+}, \mathrm{Mg}^{2+}, \mathrm{K}^{+}, \mathrm{Na}^{+}\right)$l'ont été par la méthode de l'acétate d'ammonium décrite par (Thomas, 1982) et le $\mathrm{pH}$ Eau a été déterminé par la méthode potentiométrique en utilisant un $\mathrm{pH}$-mètre.

\section{Evaluation des rendements}

Le matériel utilisé est la balance de portée $5 \mathrm{~kg}$ ayant servi à la pesée du paddy obtenu à la récolte dans le carré de rendement installé sur chacune des parcelles élémentaires. En effet, deux semaines après le repiquage des plants de riz, des carrés de densité sont posés au niveau de chacune des parcelles élémentaires. Cette opération a consisté à délimiter sur chaque parcelle élémentaire, $1 \mathrm{~m}^{2}$ de parcelle où la densité des plants est la plus forte possible. A la fin du cycle, ces carrés de densité sont devenus les carrés de rendement au moment de la récolte et tous les produits issus de ces carrés sont systématiquement pesés. La mesure du rendement n'a pas pris en compte le nombre de panicules par plante, ni celui de talles/pied et ni celui du nombre de grains par panicule.

\section{Traitement statistique des données}

L'analyse de la variance a été effectuée sous le logiciel R-3.4.1 pour tester les effets des différents facteurs (labour, bloc et campagne) au regard des différents rendements obtenus ainsi que des teneurs des principaux 
paramètres physico-chimiques durant les trois campagnes successives. Enfin, une Analyse en Composantes Principales (ACP) en utilisant le package FactoMineR dans le logiciel R, a permis d'étudier les liens existant entre le rendement et les paramètres physico-chimiques analysés.

\section{Resultats}

\section{Effets du labour sur le statut nutritif du sol}

Les effets du labour sur le statut nutritif du sol sont analysés par traitement et par campagne. Les résultats des campagnes 2014, 2015 et 2016 sont présentés dans les tableaux 1 et 2 :

Tableau 1: Statut nutritif du sol par campagne et traitement

\begin{tabular}{ccccccccccc}
\hline \multirow{2}{*}{ Paramètre } & $\begin{array}{c}\text { Av } \\
\text { Essai }\end{array}$ & \multicolumn{1}{c}{ C 1 } & \multicolumn{1}{c}{ C 2} & & & C 3 \\
& $\begin{array}{c}\text { Etat } \\
\text { initial }\end{array}$ & $\begin{array}{c}\text { Sans } \\
\text { Lab }\end{array}$ & $\begin{array}{c}\text { Lab } \\
\text { Man }\end{array}$ & $\begin{array}{c}\text { Lab } \\
\text { Méc }\end{array}$ & $\begin{array}{c}\text { Sans } \\
\text { Lab }\end{array}$ & $\begin{array}{c}\text { Lab } \\
\text { Man }\end{array}$ & $\begin{array}{c}\text { Lab } \\
\text { Méc }\end{array}$ & $\begin{array}{c}\text { Sans } \\
\text { Lab }\end{array}$ & $\begin{array}{c}\text { Lab } \\
\text { Man }\end{array}$ & $\begin{array}{c}\text { Lab } \\
\text { Méc }\end{array}$ \\
\hline Argile & 53,23 & 51,92 & 54,27 & 55,31 & 50,47 & 54,39 & 55,51 & 49,78 & 53,88 & 55,22 \\
\hline Limon & 39,83 & 38,93 & 41,16 & 40,41 & 37,91 & 39,42 & 39,27 & 37,56 & 40,37 & 39,53 \\
\hline Sable & 6,58 & 7,97 & 4,30 & 3,82 & 7,87 & 5,2 & 4,2 & 10,32 & 5,15 & 4,57 \\
\hline C\% & 1,93 & 1,37 & 2,19 & 2,83 & 1,11 & 2,33 & 3,08 & 1,13 & 2,33 & 2,94 \\
\hline N\% & 0,17 & 0,14 & 0,19 & 0,22 & 0,11 & 0,20 & 0,21 & 0,12 & 0,18 & 0,20 \\
\hline C/N & 11,35 & 10,16 & 11,36 & 13,07 & 10,26 & 11,90 & 14,66 & 9,13 & 12,93 & 14,45 \\
\hline M.O.\% & 3,32 & 2,35 & 3,77 & 4,87 & 1,91 & 4,01 & 5,29 & 1,94 & 4,00 & 5,05 \\
\hline Ca ++ & 2,07 & 1,72 & 2,99 & 3,61 & 1,53 & 2,1 & 2,90 & 1,63 & 2,10 & 3,17 \\
\hline Mg++ & 1,46 & 1,31 & 2,66 & 3,06 & 1,30 & 2,07 & 2,87 & 0,82 & 2,65 & 3,39 \\
\hline K+ & 1,27 & 1,10 & 2,11 & 2,19 & 0,93 & 1,43 & 2,48 & 0,91 & 1,53 & 2,3 \\
\hline Na+ & 1,8 & 1,61 & 2,73 & 2,98 & 1,42 & 1,80 & 2,83 & 1,77 & 1,93 & 2,06 \\
\hline$\sum$ cations & 6,6 & 5,74 & 10,48 & 11,83 & 5,19 & 7,40 & 11,08 & 5,12 & 8,21 & 10,91 \\
\hline CEC & 30,63 & 28,89 & 32,43 & 35,60 & 26,68 & 30,58 & 33,08 & 25,3 & 29,08 & 32,23 \\
\hline Pass. Ppm & 25,55 & 23,50 & 26,27 & 29,54 & 21,49 & 24,72 & 28,74 & 22,1 & 25,96 & 27,50 \\
\hline pH Eau & 5,09 & 4,93 & 5,07 & 5,15 & 4,96 & 5,04 & 5,15 & 4,7 & 5,02 & 5,13 \\
\hline
\end{tabular}

\section{Légende :}

Sans Lab $=$ Sans labour $;$ Lab Man $=$ Labour Manuel $;$ Lab Méc $=$ Labour Mécanisé et Ci : $\mathrm{i}^{\mathrm{ème}}$ campagne

$\underline{\text { Tableau } 2}$ : Bilan de l'évolution des teneurs du sol $(0-40 \mathrm{~cm})$ en Argile, Limon, Sable, C, N, $\mathrm{MO}, \mathrm{C} / \mathrm{N}, \mathrm{Ca}++, \mathrm{Mg}++, \mathrm{K}^{+}, \mathrm{Na}+, \sum \mathrm{Cat}, \mathrm{CEC}$, Pass et $\mathrm{pH}$ Eau sur les parcelles soumises aux traitements Sans labour, Labour manuel et Labour Mécanisé au cours des campagnes C1, $\mathrm{C} 2$ et $\mathrm{C} 3$

\begin{tabular}{cccccccccc}
\hline & \multicolumn{2}{c}{$\begin{array}{c}\text { Taux d'évolution en } \\
\text { \% sous Sans Lab }\end{array}$} & \multicolumn{2}{c}{$\begin{array}{c}\text { Taux d'évolution en } \\
\text { \% sous Lab Man }\end{array}$} & \multicolumn{3}{c}{$\begin{array}{c}\text { Taux d'évolution } \\
\text { en \% sous Lab Méc }\end{array}$} \\
\cline { 2 - 11 } & C1 & C2 & C3 & C1 & C2 & C3 & C1 & C2 & C3 \\
\cline { 2 - 12 } & & & & & & & & & \\
\hline Argile & $-2,5$ & -5 & $-6,5$ & 2 & 2,2 & 1,2 & 3,9 & 4,3 & 3,7 \\
\hline Limon & $-2,3$ & -5 & $-5,7$ & 3,3 & -1 & 1,4 & 1,5 & $-1,4$ & $-0,8$ \\
\hline
\end{tabular}




\begin{tabular}{cccccccccc}
\hline Sable & 21 & 20 & 57 & $-34,7$ & -21 & $-21,7$ & $-41,9$ & $-36,2$ & $-30,5$ \\
\hline $\mathrm{C}(\%)$ & -29 & -42 & -41 & 13,5 & 20,7 & 20,7 & 46,6 & 59,6 & 52,3 \\
\hline $\mathrm{N}(\%)$ & -18 & -35 & -29 & 0,12 & 17,6 & 5,9 & 29,4 & 23,5 & 17,6 \\
\hline $\mathrm{C} / \mathrm{N}$ & -10 & -10 & -20 & $10^{-3}$ & 4,8 & 13,9 & 15,2 & 29,2 & 27,3 \\
\hline $\mathrm{MO}(\%)$ & -29 & -42 & -42 & 0,14 & 20,8 & 20,5 & 46,7 & 59,3 & 52,1 \\
\hline $\mathrm{Ca}++$ & -17 & -26 & -21 & 0,44 & 1,4 & 1,4 & 74,4 & 40,1 & 53,1 \\
\hline $\mathrm{Mg}++$ & -10 & -11 & -44 & 0,82 & 41,8 & 81,5 & 110 & 96,6 & 132,2 \\
\hline $\mathrm{K}+$ & -13 & -27 & -28 & 0,66 & 12,6 & 20,5 & 72 & 95,3 & 81,1 \\
\hline $\mathrm{Na}+$ & -11 & -21 & -2 & 0,517 & 0 & 7,2 & 66 & 57,2 & 14,4 \\
\hline$\sum \mathrm{Cat}(\mathrm{méq} / 100 \mathrm{~g})$ & -13 & -21 & -22 & 0,588 & 12,1 & 24,4 & 79,2 & 67,9 & 65,3 \\
\hline $\mathrm{CEC}(\mathrm{méq} / 100 \mathrm{~g})$ & -6 & -13 & -17 & 0,059 & $-0,2$ & $-5,1$ & 16,2 & 8 & 5,2 \\
\hline Pass (ppm) & -8 & -16 & -14 & 0,028 & $-3,2$ & 1,6 & 15,6 & 12,5 & 7,6 \\
\hline $\mathrm{pH} \mathrm{Eau}$ & -3 & -3 & -8 & $-0,004$ & -1 & $-1,4$ & 1,2 & 1,2 & 0,8 \\
\hline
\end{tabular}

Légende :

Sans Lab $=$ Sans labour $;$ Lab Man = Labour Manuel $;$ Lab Méc $=$ Labour Mécanisé et Ci : $\mathrm{i}^{\mathrm{ème}}$ campagne

D'une manière générale, l'analyse de la granulométrie a montré (tableaux 1 et 2) que sur les sols labourés ont enregistré une réduction de la proportion de sable (21 à 34,7\% en labour manuel et 30,5 à 41,9\% en labour mécanisé) tandis que c'est le contraire qui a été remarqué au niveau de la parcelle témoin (Sans labour). Spécifiquement, par traitement et campagne, il ressort ce qui suit :

Pendant chaque campagne d'étude, les parcelles non labourées (sans labour) ont vu les teneurs des paramètres analysés régresser en dehors du sable. Ainsi, l'argile et le limon ont enregistré des taux négatifs progressifs comme le $\mathrm{Mg}++$, le $\mathrm{K}+$, la $\sum$ Cat et la CEC tandis que le reste a connu une régression négative en dents de scie. En clair, lorsque les parcelles ne sont pas labourées avant le semis, l'état structural du sol se trouve fortement affecté avec par exemple la forte élévation enregistrée pour la teneur du sable ( $21 \%$ en $\mathrm{C} 1$ contre $20 \%$ en $\mathrm{C} 2$ et $57 \%$ en $\mathrm{C} 3$ ).

Sous les parcelles labourées à la houe (labour manuel), l'évolution a été positive chaque année pour la plupart des paramètres tant physiques que chimiques à la différence du limon $(-1 \%)$ en $\mathrm{C} 2$ et le sable ayant enregistré des taux négatifs au cours des 3 campagnes $(\mathrm{C} 1, \mathrm{C} 2$ et $\mathrm{C} 3$ ) pour respectivement $-34,7 \%,-21 \%$ et $-21,7 \%$. Il faut signaler qu'au cours des 3 années de culture, l'évolution a été en dents de scie pour l'argile, le limon et le $\mathrm{pH}$. Elle est positive et progressive pour le $\mathrm{N}$, le $\mathrm{C} / \mathrm{N}$, le $\mathrm{Mg}++$ et la $\sum$ Cat lors des 3 campagnes mais positive en $\mathrm{C} 1$ pour la $\mathrm{CEC}$ et négative en $\mathrm{C} 2$ et $\mathrm{C} 3$ dégressivement. Enfin, le taux d'évolution a été positif de $\mathrm{C} 1$ à $\mathrm{C} 2$ pour le $\mathrm{C}$ et inchangé en $\mathrm{C} 3$ tandis qu'il a été positif en dents de scie pour le $\mathrm{Na}+$. 
Il ressort de ces résultats que le labour à la houe a impacté positivement l'état structural du sol du point de vue de sa teneur en sable qui a sensiblement diminué lors de chaque campagne au profit de l'augmentation de celle en argile. Il en est de même du rapport $\mathrm{C} / \mathrm{N}$, de la $\mathrm{MO}$ et de la $\sum$ Cat.

Pour les parcelles labourées avec le rotavator (labour mécanisé), le taux d'évolution a été positif de façon générale par campagne et ce pour les paramètres chimiques et l'argile. En C1, l'évolution a été positive pour le limon $(1,5 \%)$ et négative en $\mathrm{C} 2$ et $\mathrm{C} 3(-1,4$ et $-0,8 \%)$ contre des taux négatifs pour le sable durant les 3 campagnes $(\mathrm{C} 1, \mathrm{C} 2$ et $\mathrm{C} 3)$ pour les valeurs respectives $-41,9,-36,2$ et $-30,5$. Sur les 3 années, le taux d'évolution a été positif en dents de scie pour l'argile, négatif et régressif pour le sable tandis qu'il est régressif et varié pour le limon. Il est positif et dégressif pour le $\mathrm{N}$, le $\mathrm{Na}+$, la $\sum$ Cat, la CEC et le Pass. Le $\mathrm{C}$, le $\mathrm{C} / \mathrm{N}$, la $\mathrm{MO}$, le $\mathrm{Ca}++$ et le $\mathrm{Na}+$ ont enregistré des taux positifs en dents de scie. Enfin le $\mathrm{pH}$ a enregistré pour la $\mathrm{C} 1$ et $\mathrm{C} 2$ le même taux positif $(1,2 \%)$ mais qui a chuté à $0,8 \%$ en $\mathrm{C} 3$. Sous ce traitement, il faut retenir qu'il y a eu une évolution perceptible au niveau de tous les paramètres par rapport à l'état initial avec une moyenne de diminution de $36 \%$ de la teneur du sable sur les 3 années de campagnes.

D'après les résultats de l'ANOVA, nous avons remarqué d'une manière générale que l'effet traitement (sans labour, labour manuel et labour mécanisé) est très hautement significatif au seuil de $0,1 \%$. En particulier, l'effet campagne est également très hautement significatif sur le sable et moins sur le limon $(0,1)$ tandis que l'interaction campagne*labour est significatif au seuil de 0,05 sur le sable et hautement significatif $(0,01)$ sur l'argile. Le constat est demeuré presque le même pour tous les autres paramètres chimiques en dehors du $\mathrm{pH}$ eau pour lequel seul le labour a été très hautement significatif. Les blocs et toutes les interactions «bloc*campagne » ou «bloc*labour» ou «bloc*campagne*labour » n'ont aucun effet significatif sur aucun paramètre. Il ressort de tout ce qui précède que le labour à travers ses trois modalités a un effet très hautement significatif sur l'état nutritif du sol mais plus particulièrement, le labour mécanisé a eu un effet très positif sur sa fertilité.

\section{Effets de la succession du labour sur le statut nutritif du sol}

Le tableau 3 ci-après présente les résultats des labours successifs sur le statut nutrif du sol.

Tableau 3 : Bilan de la succession du labour sur le statut nutritif du sol

\begin{tabular}{|c|c|c|c|c|c|c|c|c|c|c|}
\hline \multirow[b]{2}{*}{$\begin{array}{l}\text { Paramètres } \\
\text { analysés }\end{array}$} & \multicolumn{3}{|c|}{ Sans Labour } & \multicolumn{3}{|c|}{ Labour manuel } & \multicolumn{3}{|c|}{ Labour mécanisé } & \multirow{2}{*}{$\begin{array}{l}\text { Normes pour } \\
\text { le riz pluvial }\end{array}$} \\
\hline & $\begin{array}{c}\text { Etat } \\
\mathrm{i}\end{array}$ & Etat $\mathrm{f}$ & $\begin{array}{c}\text { Evol. } \\
\%\end{array}$ & $\begin{array}{c}\text { Etat } \\
\mathrm{i}\end{array}$ & Etat $\mathrm{f}$ & $\begin{array}{c}\text { Evol. } \\
\%\end{array}$ & $\begin{array}{c}\text { Etat } \\
\mathrm{i}\end{array}$ & Etat $\mathrm{f}$ & $\begin{array}{c}\text { Evol. } \\
\%\end{array}$ & \\
\hline Argile & 53,2 & 49,8 & $-6,5$ & 53,2 & 53,9 & 1,2 & 53,2 & 55,2 & 3,7 & \\
\hline
\end{tabular}




\begin{tabular}{ccccccccccc}
\hline Limon & 39,8 & 37,6 & $-5,7$ & 39,8 & 40,4 & 1,4 & 39,8 & 39,5 & $-0,8$ & \\
\hline Sable & 6,6 & 10,3 & 56,8 & 6,6 & 5,2 & $-21,7$ & 6,6 & 4,8 & $-30,6$ & \\
\hline $\mathrm{C}(\%)$ & 1,9 & 1,1 & $-41,5$ & 1,9 & 2,3 & 20,7 & 1,9 & 2,9 & 52,3 & $>0,8$ \\
\hline $\mathrm{N}(\%)$ & 0,2 & 0,1 & $-29,4$ & 0,2 & 0,2 & 5,9 & 0,2 & 0,2 & 17,6 & $2<\mathrm{N}<0,03$ \\
\hline $\mathrm{C} / \mathrm{N}$ & 11,4 & 9,1 & $-19,6$ & 11,3 & 12,9 & 13,9 & 11,4 & 14,5 & 27,3 & $8<\mathrm{C} / \mathrm{N}<15$ \\
\hline $\mathrm{MO}(\%)$ & 3,3 & 1,9 & $-41,6$ & 3,3 & 4 & 20,5 & 3,3 & 5,1 & 52,1 & $>2$ \\
\hline $\mathrm{Ca}++$ & 2,1 & 1,6 & $-21,3$ & 2,1 & 2,1 & 1,5 & 2,1 & 3,2 & 53,1 & 3,5 \\
\hline $\mathrm{Mg}++$ & 1,5 & 0,8 & $-43,8$ & 1,5 & 2,7 & 81,5 & 1,5 & 3,4 & 132,2 & 1,5 \\
\hline $\mathrm{K}+$ & 1,27 & 0,9 & $-28,4$ & 1,3 & 1,5 & 20,5 & 1,3 & 2,3 & 81,1 & $0,4<\mathrm{K}+<0,8$ \\
ou $>0,8$ \\
\hline $\mathrm{Na}+$ & 1,8 & 1,8 & $-1,7$ & 1,8 & 1,9 & 7,2 & 1,8 & 2,1 & 14,4 & $>0,4$ \\
\hline $\begin{array}{c}\sum \mathrm{Cat} \\
(\mathrm{Méq} / 100 \mathrm{~g})\end{array}$ & 6,6 & 5,1 & $-22,4$ & 6,6 & 8,2 & 24,4 & 6,6 & 10,9 & 65,3 & \\
\hline $\begin{array}{c}\mathrm{CEC} \\
(\mathrm{méq} / 100 \mathrm{~g})\end{array}$ & 30,6 & 25,3 & $-17,4$ & 30,6 & 29,1 & $-5,1$ & 30,6 & 32,2 & 5,2 & $20<\mathrm{CEC}<40$ \\
\hline Pass (ppm) & 25,6 & 22,1 & $-13,5$ & 25,6 & 26 & 1,6 & 25,6 & 27,5 & 7,6 & $>20$ \\
\hline $\begin{array}{c}\text { pH Eau } \\
5,1\end{array}$ & 4,7 & $-7,7$ & 5,1 & 5,0 & $-1,4$ & 5,1 & 5,1 & 0,8 & $\begin{array}{c}\text { Supporte des } \\
\text { pH de 4 à } 8\end{array}$ \\
\hline
\end{tabular}

\section{Légende :}

Etat $\mathrm{i}=$ Etat initial $;$ Etat $\mathrm{f}=$ Etat final $;$ Evol. $=$ Evolution

Du tableau 3, il ressort que :

Sous le traitement «Sans labour» une nette régression des teneurs de tous les paramètres entre l'état initial et l'état final. En effet les différences entre les valeurs initiales de tous les paramètres avant les essais et celles obtenues à la fin des essais en 2016 après les campagnes $\mathrm{C} 1, \mathrm{C} 2$ et $\mathrm{C} 3$ sont négatives. Ceci démontre d'une perte de fertilité de la parcelle initiale ayant reçu le traitement «Sans labour» au cours des 3 années de production ;

- $\quad$ Les résultats sont presque différents sous le labour manuel. En effet, la régression entre les deux status (final et initial) n'a été notée qu'au niveau du sable $(-0,22 \%)$, de la CEC $(-0,05 \%)$ et du pH Eau (-0,01\%). Pour tous les autres paramètres tant physiques que chimiques, le taux d'évolution a été positif ; ce qui montre une légère amélioration de la fertilité en dépit des régressions obtenues pour la $\mathrm{CEC}$ et le $\mathrm{pH}$ Eau ;

Enfin, sous le traitement «Labour mécanisé», les deux valeurs négatives enregistrées pour l'évolution concernent le limon $(-0,01 \%)$ et le sable (-0,31\%). Le $\mathrm{Mg}++$, le $\mathrm{K}+$, la $\sum \mathrm{Cat}$, le $\mathrm{Ca}++$, le $\mathrm{C}$ et la $\mathrm{MO}$ présentent les taux d'évolution les plus élevés ( $>50 \%$ ) ; par exemple 52\% pour la MO. Dans l'ensemble, le bilan enregistré avec le labour mécanisé illustre un gain de fertilité car le taux de régression du sable et celui du limon ne l'ont pas affecté de façon sensible. 
Des résultats de l'ANOVA, nous pouvons déduire que la succession des campagnes (effet campagne) a été très hautement significative sur le sable et non significative sur le reste des paramètres.

\section{Effets du labour sur le rendement}

Le tableau 4 ci-après présente les résultats relatifs aux effets des différents modes de labour sur le rendement du sol.

Tableau 4 : Evolution du rendement moyen du riz par campagne et traitement

\begin{tabular}{ccccccccc}
\hline & C 1 & \multicolumn{3}{c}{ C 2 } & & C 3 & \\
\hline Sans & Lab & Lab & Sans & Lab & Lab & Sans & Lab & Lab \\
Lab & Man & Méc & Lab & Man & Méc & Lab & Man & Méc \\
\hline 1,16 & 3,32 & 4,47 & 1,04 & 3,44 & 4,71 & 0,89 & 3,40 & 4,73 \\
\hline
\end{tabular}

Légende :

Sans Lab $=$ Sans labour $;$ Lab Man $=$ Labour Manuel $;$ Lab Méc $=$ Labour Mécanisé et Ci : $i^{\text {ème }}$ campagne

Du tableau 4, il ressort ce qui suit :

- $\quad$ Les rendements obtenus pour le riz paddy au cours des 3 campagnes par traitement sont plus faibles sous le traitement témoin «sans labour»;

- $\quad$ De la campagne 1 à la campagne 3, le rendement a connu une régression progressive sous le traitement «sans labour» avec respectivement pour valeurs 1,16 th/ha, 1,04 t/ha et $0,89 \mathrm{t} / \mathrm{ha}$;

- $\quad$ Avec le labour manuel, les rendements ont presque triplé en $\mathrm{C} 1$ et en $\mathrm{C} 2$ et presque quadruplé ceux obtenus en $\mathrm{C} 3$ sous «sans labour». Mais concrètement, avec le labour manuel, le rendement n'a pas trop varié de $\mathrm{C} 1$ à $\mathrm{C} 3$ où d'ailleurs il a légèrement chuté $(3,40 \mathrm{t} / \mathrm{ha}$ en $\mathrm{C} 3$ contre $3,44 \mathrm{t} / \mathrm{ha}$ en $\mathrm{C} 2$ et $3,32 \mathrm{t} / \mathrm{ha}$ en $\mathrm{C} 1$ ).

- $\quad$ Pour le labour mécanisé, les rendements obtenus sont les meilleurs et conformes à la norme qui est de 4 à 5 t/ha. En effet, au cours des 3 campagnes $\mathrm{C} 1, \mathrm{C} 2$ et $\mathrm{C} 3$, les valeurs obtenues sont respectivement $4,47 \mathrm{t} / \mathrm{ha}$, $4,71 \mathrm{t} / \mathrm{ha}$ et $4,73 \mathrm{t} / \mathrm{ha}$.

Enfin, d'après l'ANOVA, seul le facteur «labour» a un effet très hautement significatif sur le rendement; ce qui montre que le type de labour impacte réellement l'augmentation ou la diminution du rendement du riz paddy. En outre, l'étude des corrélations entre le rendement et le statut nutritif du sol a bien montré (Figure 1 et Tableau 5) qu'il y a une forte dépendance entre certains paramètres étudiés et les rendements obtenus en labour mécanisé. 


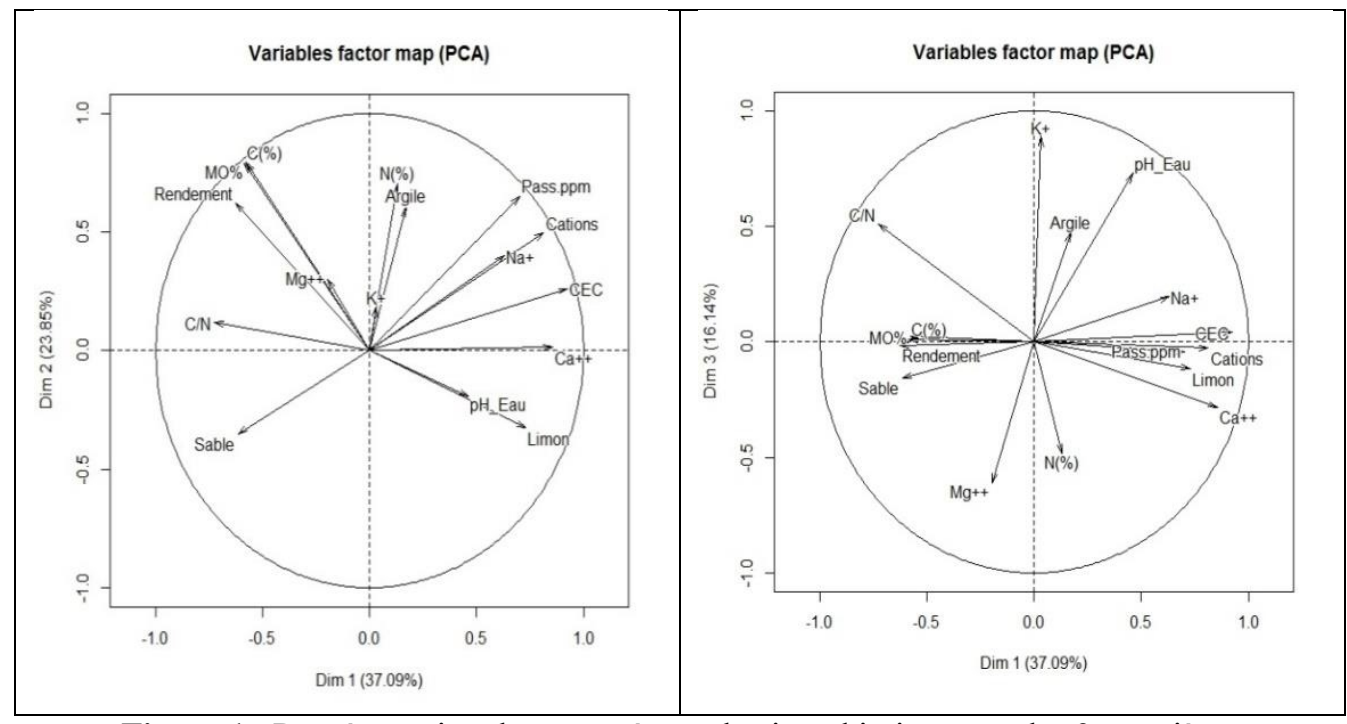

$\underline{\text { Figure } 1}$ : Représentation des paramètres physico-chimiques sur les 3 premières composantes principales

Afin de décrire les liens entre le rendement et les paramètres de fertilité du sol, une analyse en Composantes Principales (ACP) montre que les deux premières composantes concentrent respectivement 37,09 et 23,85 $\%$, soit au total $60,94 \%$ des variations du tableau de départ ce qui est suffisant pour garantir une précision d'interprétation. Cependant, l'analyse des corrélations indique que les variables $\mathrm{Mg}++, \mathrm{K}+, \mathrm{pH}$ eau ne sont prise en compte par aucune de ces deux premières composantes principales mais plutôt par la troisième. De ce fait, les trois premières composantes principales, regroupant $77,41 \%$ des variations de départ sont retenues pour décrire les liens entre le rendement et les paramètres physico-chimiques issues du labour mécanisé.

L'analyse du tableau de corrélation révèle qu'une augmentation des paramètres tels que : Sable, $\mathrm{C}(\%), \mathrm{C} / \mathrm{N}, \mathrm{MO} \%, \mathrm{~N}(\%)$, Argile d'une part (Dim. 1 et Dim.2) et d'autre part, C(\%), MO\%, $\sum$ Cat (respectivement Limon, $\mathrm{Na}+, \sum \mathrm{Cat}, \mathrm{CEC}$ et Pass.ppm) induit une augmentation (respectivement diminution) du rendement agricole tandis que les paramètres $\mathrm{Mg}++, \mathrm{K}+$ et $\mathrm{pH}$ Eau (Dim.3) n'ont aucun effet sur le rendement. Les paramètres $\sum$ Cat et Pass jouent des rôles ambigus.

Les résultats des corrélations entre les variables de départ et chacune des trois premières composantes principales sont présentés au tableau 5.

$\underline{\text { Tableau } 5}$ : Corrélations entre les variables de départ et chacune des trois premières composantes principales

\begin{tabular}{cccc}
\hline & Dim.1 & Dim.2 & Dim.3 \\
\cline { 2 - 4 } Argile & 0.17 & 0.61 & 0.47 \\
\hline Limon & 0.73 & -0.33 & -0.12 \\
\hline
\end{tabular}




\begin{tabular}{cccc}
\hline Sable & -0.61 & -0.35 & -0.16 \\
\hline $\mathrm{C}(\%)$ & -0.59 & 0.79 & 0.01 \\
\hline $\mathrm{N}(\%)$ & 0.13 & 0.7 & -0.48 \\
\hline $\mathrm{C} / \mathrm{N}$ & -0.73 & 0.12 & 0.51 \\
\hline $\mathrm{MO} \%$ & -0.58 & 0.79 & 0.02 \\
\hline $\mathrm{Ca}++$ & 0.85 & 0.01 & -0.28 \\
\hline $\mathrm{Mg}++$ & -0.2 & 0.3 & -0.61 \\
\hline $\mathrm{K}+$ & 0.03 & 0.18 & 0.89 \\
\hline $\mathrm{Na}+$ & 0.63 & 0.4 & 0.2 \\
\hline$\sum$ Cations & 0.81 & 0.5 & -0.03 \\
\hline $\mathrm{CEC}$ & 0.92 & 0.26 & 0.04 \\
\hline Pass.ppm & 0.7 & 0.65 & -0.04 \\
\hline pH_Eau & 0.46 & -0.19 & 0.73 \\
\hline Rendement & -0.63 & 0.62 & -0.02 \\
\hline
\end{tabular}

\section{Effets de la succession du labour sur le rendement}

Des résultats obtenus et conformément aux données illustrées par les tableaux 1, 2, 3 et 4, nous pouvons dire que la succession du labour a eu des effets sur les parcelles. En effet, la parcelle de l'état initial à l'état final sous le traitement «Sans Labour» a eu tous ses paramètres modifiés (chute des valeurs) ; ce qui est préjudiciable à l'état de fertilité du sol. En revanche, au niveau des parcelles ayant reçu le labour, la plupart des teneurs des paramètres physico-chimiques ont connu d'augmentation sous le labour manuel tandis qu'en labour mécanisé, tous les paramètres chimiques ont eu des teneurs élevées à la $\mathrm{C} 3$. Le constat a été presque le même pour les rendements qui n'ont pas décru au fil des campagnes mais au contraire ont augmenté ou n'ont presque pas varié comme c'est le cas sous le labour manuel au cours de $\mathrm{C} 2$ et $\mathrm{C} 3$.

Le cas des parcelles «Sans labour » indique le rôle primordial que joue le labour dans l'amélioration de l'état nutritif du sol fut-il manuel ou mécanisé. En effet au cours des essais, les cultures n'ont fait que puiser dans le stock d'éléments nutritifs disponibles dans le sol depuis l'état initial à l'état final pour subvenir à leurs besoins tout en l'appauvrissant. C'est ce qui justifie le rendement moyen d'1 ha obtenu durant les 3 campagnes sur les parcelles non labourées.

\section{Discussion}

Les résultats obtenus pour les paramètres chimiques des sols labourés sont conformes aux normes d'ORSTOM (1980) tandis que la plupart de ceux obtenus à partir des sols non labourés sont en-deçà. Il en est de même des paramètres physiques des sols étudiés lorsque nous prenons en compte les travaux de Azontonde et Verheye (1986) et ceux de Baize (1988). Il apparaît donc que le labour a montré de façon irréfutable qu'il améliore les paramètres physico-chimiques du sol de manière à le rendre plus fertile 
tandis que le non labour l'expose à son appauvrissement lorsqu'il est cultivé dans les conditions de l'essai c'est-à-dire sans cultures de couvertures de sol et sans apports de résidus ou pratique de mulching. C'est ainsi que le travail du sol dans son ensemble contribue à la diminution du taux de sable c'est-àdire à l'augmentation de celui de l'argile par la fissuration des structures massives, des semelles compactes et des croûtes ; ce qui permet d'augmenter la porosité et de favoriser le développement des racines. En outre, il permet également le mélange des engrais chimiques et organiques au profil. Ces résultats confirment ceux de Güclü et al., (2002) qui ont montré que ce sont la masse volumique du sol et sa résistance à la pénétration qui sont les plus modifiées par les outils du travail de sol. Le travail à la houe s'est traduit par le sectionnement de la structure et le retournement en bloc du profil. Cet état du sol labouré à la houe est plus dense impliquant des réserves en eau limitées par rapport au travail du sol à l'aide d'un rotavator. Quant à ce dernier agissant par sectionnement et par choc, il réalise une structure meuble formée de particules de terre fine plaquées les unes contre les autres et mélangées avec des mottes de petites tailles dans une proportion assez réduite. Cette structure résulte du fait d'un émiettement modéré du sol suite à la rotation à vitesse moyenne $(200 \mathrm{tr} / \mathrm{mn})$ du rotor par rapport à l'avancement $\mathrm{du}$ motoculteur $(3 \mathrm{~km} / \mathrm{h})$. D'après Biffin et al., (1990), les pièces travaillantes des outils provoquent des modifications dans la dimension et la forme des fragments de terre, et améliorent la porosité et la cohésion. Ce résultat est en phase avec celui de Viltox (1985) selon qui, la rotation à vitesse élevée des outils commandés par prise de force conduit à un émiettement notable du sol sur la profondeur travaillée.

Certes les labours (manuel ou mécanisé) ont eu des effets positifs sur la fertilité du sol voire sur les rendements obtenus mais analysés dans sa globalité, ils sont loin de justifier à eux seuls l'accroissement des rendements. D'autres déterminants comme le climat, la qualité des semences et l'entretien des cultures participent aussi fortement à cette variation des rendements. Pour ce qui est des essais réalisés, seule la maîtrise du climat ne saurait être garantie, ni acquise d'avance. Mais le constat fait par FAO (2016) et selon laquelle, la mécanisation permet, d'accroître plus facilement la production de produits à plus forte valeur tout en éliminant la pénibilité physique liée aux pratiques agricoles fondées sur l'énergie musculaire corrobore bien les résultats obtenus.

D’une manière générale, la succession de la production n'a eu des répercussions négatives que sur la parcelle témoin où l'état de fertilité a réellement chuté à cause surtout du manque de la matière organique et de la perte du taux d'argile et de limon au détriment du gain de sable. Cet état de chose a certainement joué contre sa capacité de rétention en eau. Par rapport aux parcelles labourées, la succession des labours (manuel et mécanisé) a 
contribué à l'amélioration sensible de la fertilité des sols et par conséquent celle du rendement du riz. En effet, selon les travaux de Gaultney et al., (1982), plus les labours se succèdent sur un sol, plus ils rendent meuble sa structure ce qui permet un gain de rendement de $25 \%$ lorsque l'itinéraire technique est respecté.

\section{Conclusion}

A l'issue de l'étude, il est apparu que le labour ainsi que sa succession ont un effet favorable sur le statut nutritif du sol et le rendement du riz pluvial dans le bas-fond. Il faut attribuer entre autres cet effet à une modification de la porosité du sol et à un bon développement des racines du riz. Les choix en matière de travail du sol, au niveau de l'exploitation, doivent donc, entre autres, tenir compte de l'effet qu'aura ou non le travail sur les racines, grâce auxquelles se fera l'alimentation en eau et en éléments minéraux. Au vu des résultats obtenus, il est aisé d'affirmer que le labour mécanisé basé sur l'attelage motoculteur + rotavator a donné de très bons résultats comparativement au labour avec la houe. En effet, le labour mécanisé a permis d'accroître les teneurs initiales des principaux éléments nutritifs analysés, d'améliorer de façon sensible la structure du sol et d'augmenter fortement le rendement du riz. L'appréciation des résultats a en outre permis de reconnaître que les parcelles témoins c'est-à-dire non labourées ont connu d'année en année une forte régression des paramètres physico-chimiques avec pour conséquence la baisse drastique du rendement du riz.

Aujourd'hui, le moment est venu pour les petits exploitants agricoles de chercher à bénéficier de quelques bienfaits de la mécanisation agricole en optant pour le labour mécanisé. Ce n'est qu'alors qu'ils se rendront compte qu'il s'agit d'un intrant essentiel qui leur permettra de booster la production, combler la pénurie en main d'œuvre et se passer de la pénibilité physique des travaux agricoles.

\section{References:}

1. Assigbe, P. (2002) : Développement participatif de technologies pour la gestion intégrée de la fertilité des sols rizicoles du Centre Bénin. In Bal, A. B. (Eds.), ADRAO-Le centre du riz pour l'Afrique et Réseau Ouest et Centre Africain du Riz (ROCARIZ) Compte rendu de la seconde revue régionale de la recherche rizicole, 65-69.

2. Azontonde, A., et Verheye, W. (1986) : Pédologie et aptitude des terres. Etude de Factibilité pour le Développement Rural Intégré de la Province du Mono, Centre National d'Agro-Pédologie, Rapport d'étude, Abomey-Calavi, Bénin, 89 p. 
3. Baize, D. (1988) : Guide des analyses courantes en pédologie. ChoixExpression-Présentation-Interprétation. Service d'Etude des Sols et de la Carte Pédologique. Institut National de la Recherche Scientifique. Paris, 1988, ISBN : 2-7380-0075-4, 172 p.

4. Biffin J., Guerif, J. et Stengel, P. (1990) : Les processus d'évolution de l'état structural du sol : quelques exemples d'études expérimentales récentes. La structure et son évolution. Dans : La structure du sol et son évolution. Laon (France): Ed. INRA Paris, 216 p.

5. FAO, (1988) : Production de légumes dans les conditions arides et semi-arides d'Afrique tropicale, Rome Série Etude FAO, production végétale et protection des végétaux $\mathrm{n}^{\circ} 89,110 \mathrm{p}$

6. FAO, (2008) : L'eau pour l'agriculture et l'énergie en Afrique : les défis du changement climatique. Ateliers régionaux. Syrte, Jamahiriya Arabe Lybienne, 15-17, Décembre 2008. www.sirtewaterandenergy.org/.../Regional-Workshops-Fr.pdf.

7. FAO, (2016) : La mécanisation agricole: un intrant essentiel pour les petits exploitants d'Afrique Subsaharienne. Gestion intégrée des cultures, Vol. 23, 61 p.

8. Gaultney, G., Krutz W., Steinhardt, G.C. and Liljedahl, J.B. (1982): Effects of subsoil compaction on corn yields. Transactions of the ASAE, Vol. 25, $\mathrm{N}^{\circ} 3$, pp. 563-569.

9. Girard C., Walter, C., Berthelin, J., Remy, J.C., et Morel J.L. (2005) : Sols et Environnement. Cours et Etudes de cas. Dunod, coll. Sciences Sup. 832 p.

10. Gonzales, L., et Umali, D. L. (1985) : Rice production systems. In International Rice Commission - Proceedings of the 16th session of the International Rice Commission. Los baños, Laguna, Philippines. FAO, Rome.

11. Güclü Yavuzcan, H., Vatandas, M., et Gürhan, R. (2002): Soil strength as affected by tillage system and wheel traffic in wheat- corn rotation in central Anatolia. Journal of Teeamechanics, Vol. 39, pp. 23-34.

12. INRAB, (2005) : Mieux produire le riz pluvial et de bas-fond. Fiche technique de production du riz, $45 \mathrm{p}$.

13. IRAT, (1984) : Les systèmes de culture du riz pluvial. Montpellier, France. $98 \mathrm{p}$.

14. Kokoye D. (2013): Etude du fonctionnement hydrologique et hydraulique du bas-fond de Zoungo en vue de sa mise en valeur. Thèse d'Ingénieur Agronome, FSA/UNB, 100 p.

15. MAEP (Ministère de l'Agriculture, de l'Elevage et de la Pêche), (2010) : Rapport du Plan Stratégique de Relance du Secteur Agricole (PSRSA) 2010-2015, Bénin, 125 p. 
16. MAEP (Ministère de l'Agriculture, de l'Elevage et de la Pêche) (2011), : Stratégie Nationale pour le Développement de la Riziculture au Bénin (SNDR). Rapport final, $30 \mathrm{p}$.

17. MAIRIE ABOMEY-CALAVI, (2012): Plan de développement communal, quinquennat 2012-2016, $125 \mathrm{p}$.

18. Mouzou, T. (1999) : Rendement des tractoristes au labour: un paramètre permettant l'équipement rationnel en matériel agricole. Journal de la Recherche Scientifique de l'Université du Bénin, Lomé Vol.3, $\mathrm{N}^{\circ} 2$, pp. $75-83$.

19. ORSTOM (1980): Normes d'interprétations des analyses chimiques. $\mathrm{N}^{\circ} 189$ bis, Paris, $6 \mathrm{p}$.

20. SCDA (Secteur Communal pour le Développement Agricole), 2009 : Rapport sur le zonage agro écologique de la commune d'AbomeyCalavi, 2009, $65 \mathrm{p}$.

21. SCDA (Secteur Communal pour le Développement Agricole), 2012 : Rapport de fin de campagne agricole 2011-2012, 2012, 80 p.

22. Seguyl, P. (1994) : Contributions à l'étude et la mise au point des systèmes de cultures en milieu réel: petit guide d'initiation à la méthode de «création-diffusion» de technologie en milieu réelrésumé de quelques exemples significatifs d'application. Montpellier, CIRAD-Ca, $191 \mathrm{p}$.

23. Sossa, B. (2001): Contribution à la mise en valeur de la plaine d'inondation du lac Toho à Kponou ( $\mathrm{S} / \mathrm{P}$ Athiémé, Département du Mono), Thèse d'Ingénieur Agronome, FSA/UNB, $84 \mathrm{p}$.

24. Vitlox, I. O. (1985) : Compaction des sols. Colloque international: le travail du sol. Faculté des Sciences Agronomiques de l'Etat de Gembloux. 